\title{
Computational and Experimental Studies into the Conformations of a Triptycene-Based Ditopic Ligand
}

\author{
Jesús Sanmartín-Matalobos, ,a, Ana M. García-Deibea, Martín Amozaa, Matilde Fondoa, \\ Antonio J. Mota ${ }^{\mathrm{b}}$, Sourav Bhowmick ${ }^{c}$, and Neeladri Das ${ }^{\mathrm{c}}$ \\ aDpto. de Química Inorgánica, Facultad de Química, Universidad de Santiago de Compostela, Avenida \\ de las Ciencias s/n, 15782 Santiago de Compostela, Spain. Fax: +34981 528073; Tel: +34 981 563100; \\ E-mail: jesus.sanmartin@usc.es \\ ${ }^{b}$ Departamento de Química Inorgánica, Facultad de Ciencias, Universidad de Granada. 18002 \\ Granada, Spain. \\ ${ }^{c}$ Department of Chemistry, Indian Institute of Technology Patna, Patna 800 013, Bihar, India.
}

\begin{abstract}
We present a combined computational and experimental study of the possible conformations adopted by a ditopic Schiff base ligand based on triptycene. We have performed DFT calculations on a Y-shaped Schiff base ligand derived from the condensation of 2,6diaminotriptycene and 2 hydroxybenzaldehyde to obtain the relative energies of their conformers anti-s-cis, syn and anti-s-trans. Since these conformations are practically isoenergetic, interconversions of conformers proceed by rotation about C-N single bonds. NMR spectroscopy shows the presence in solution of the syn conformer, which is stable at room temperature on the NMR time scale.
\end{abstract}

Keywords: DFT calculations / Triptycene / Schiff base / Conformational isomerism

\section{INTRODUCTION}

In recent years, triptycenes, which are 9,10-dihydro-9,10-[1,2] benzenoanthracene derivatives, have attracted considerable attention because of their potential for preparing porous materials [1], since the rigid three-bladed geometry produces a $120^{\circ}$ angle between the arenes, which hinders their efficient packing in the solid state, leading to void spaces in the clefts between the arenes [2]. This characteristic structural feature of triptycenes has been also probed as fluorescent chemosensors [3,4]. Recently, Das et al. have reported both the fluorescence quenching of triptycene-based polymers by host-guest interaction with fullerene [5], as well as the first example of a truly metallasupramolecular cage based on a triptycene tecton [6].

Considering the versatile applications of triptycene derivatives, we have the intention of extending our investigations to the coordination abilities and of a triptycene polycycle, with two $\mathrm{N}, \mathrm{O}$-donor sites linked to the 2 and 6 positions (Scheme 1). This asymmetry provides chirality, so the ligand here studied is a $\left(R, R^{\prime} ; S, S^{\prime}\right)$ racemate. In this occasion, we will characterise the ligand, and will explore the possible conformations adopted by this ditopic Schiff base ligand, in an attempt to comprehend their implication in its potential coordinating behaviour, so some molecular modelling will be also presented. 


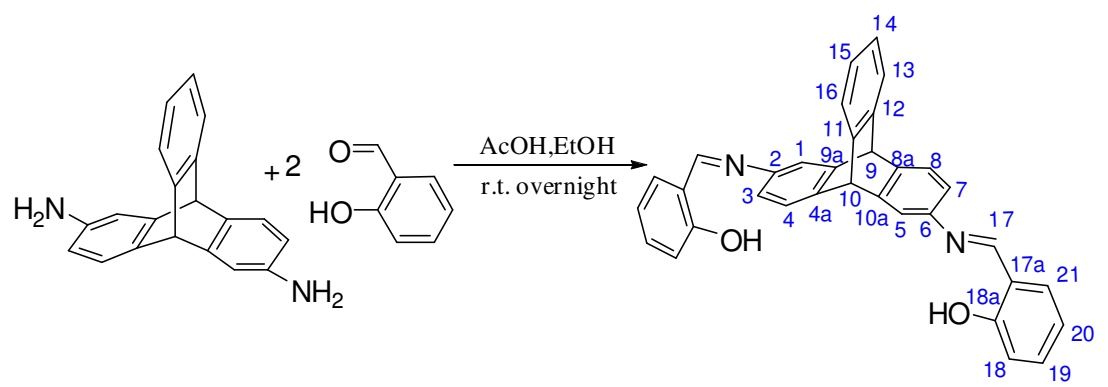

Scheme 1. Formation of the $S, S^{\prime}$ isomer of the Y-shaped Schiff base ligand, $\mathrm{H}_{2} \mathrm{~L}$, including the numbering scheme for NMR studies.

\section{RESULTS AND DISCUSSION}

The extended conjugation between the rigid three-bladed triptycene hub and both salicylaldimine pendants should lead to an Y-shaped spatial arrangement, with a $c a .120^{\circ}$ angle between the two pendants, as they should be conjugated with their corresponding arene rings. This conjugation can also hinder a free rotation around the $\mathrm{C}-\mathrm{N}$ single bond of the attachment to the corresponding fused ring.

With these premises in mind, only three conformational isomers have been considered for the theoretical calculations, all of them with an $E$-configuration for the azomethine group, as this is typically stabilised by an intramolecular $\mathrm{O}_{\text {phenol }}-\mathrm{H} \cdots \mathrm{N}_{\text {imine }}$ bonding [7].

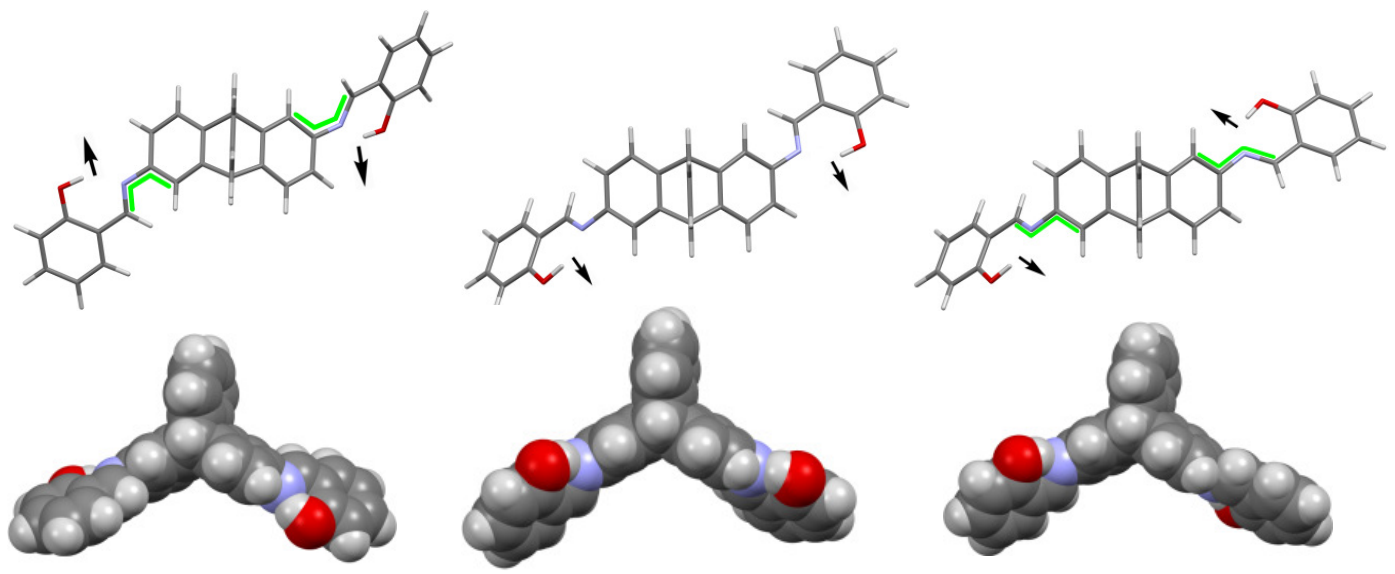

Fig. 1. Two different views of the anti-s-cis (left), syn (centre) and anti-s-trans (right) conformational isomers of $\mathrm{H}_{2} \mathrm{~L}$ obtained by DFT energy-minimisation. Only $R, R^{\prime}$ isomers are represented. Arrows are indicting the orientation of the $\mathrm{N}, \mathrm{O}$-donor sets for chelation.

These conformers have been qualified as syn or anti, in light of the orientation of the two $\mathrm{N}, \mathrm{O}$-donor sets, either to the same or to opposite sides, respect to the triptycene residue, as Fig.1 shows. Since the ditopic ligand shows a $C_{2 \mathrm{v}}$ point group symmetry, for an anti configuration of the donor domains, two other conformers are possible, so the attachment of the imine bond to the triptycene hub show a s-cis or a s-trans disposition [8]. Thus, those $\mathrm{H}$ atoms in positions 1 and 5, or 3 and 7, respectively, are in close proximity to both azomethine-H atoms. Fig. 1 shows the calculated conformational isomers of $\mathrm{H}_{2} \mathrm{~L}$ obtained by DFT energy-minimisation. The ligand 
symmetry leads to the two central triptycene $C$ atoms, at positions 9 and 10, to be asymmetric, but we have only used the $R, R^{\prime}$ enantiomer to represent the conformers.

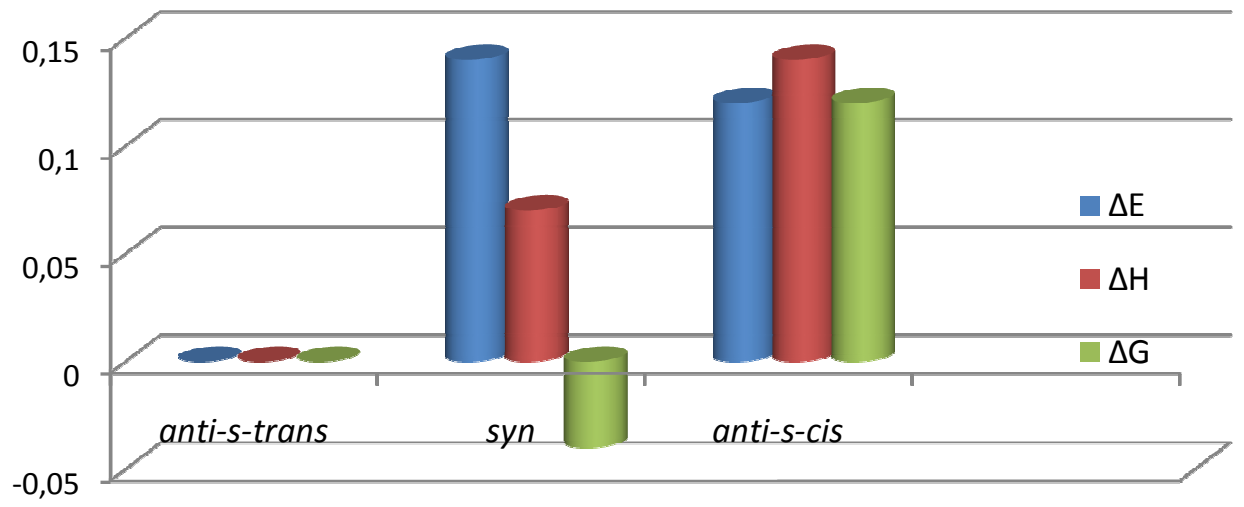

Fig. 2. Energy profile for the three conformers studied for $\mathrm{H}_{2} \mathrm{~L}$ from DFT energy-minimisations.

DFT studies indicate that these three conformers are practically isoenergetic, as Fig. 2 shows, and this is explained with very low energy barriers for the transition states, where the salicylaldimine branches are slightly twisted.

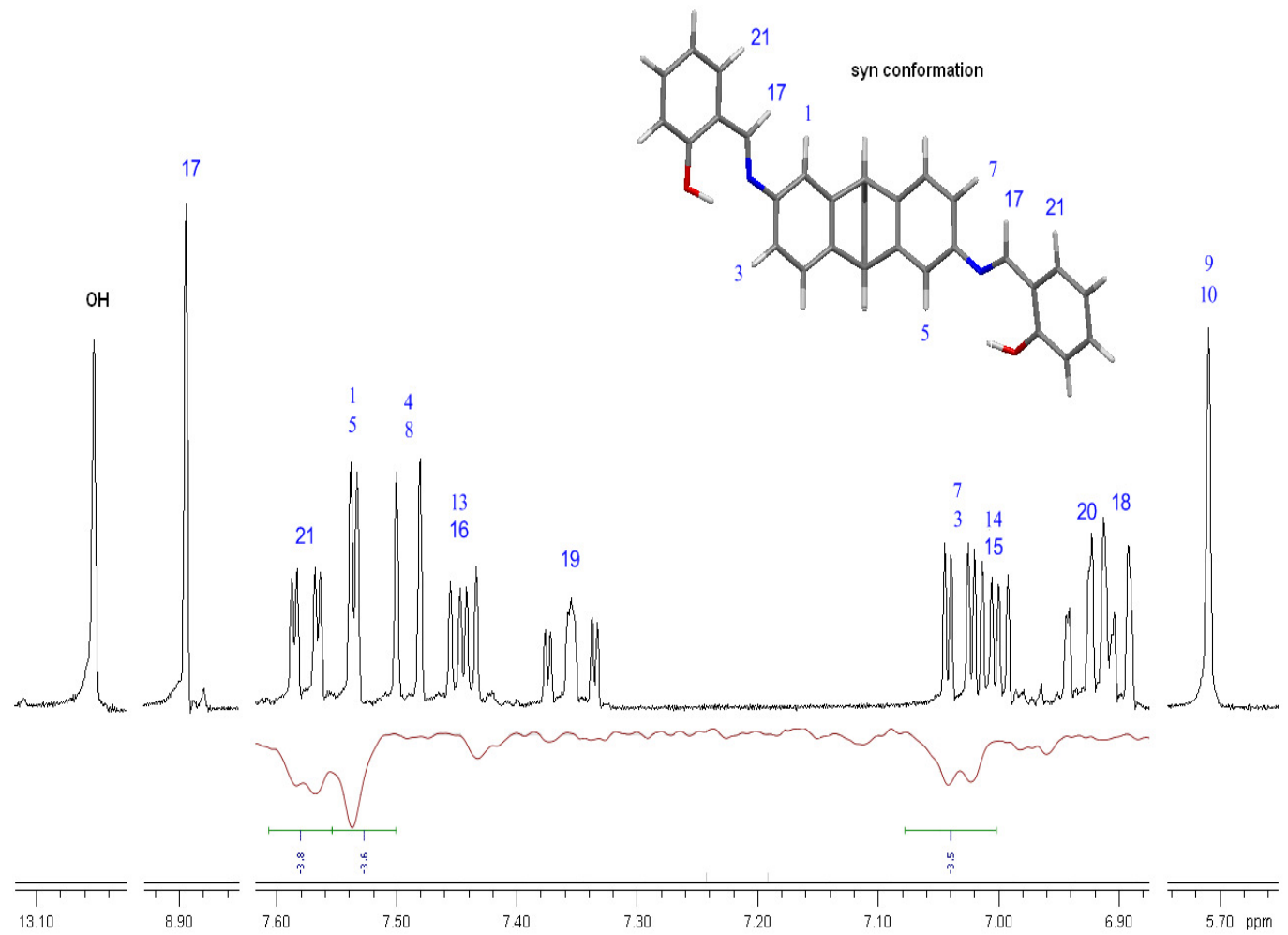

Fig. 3. Molecular structure of $\mathrm{H}_{2} \mathrm{~L}$ determined using a combination of NMR spectroscopic methods (COSY, HMQC, HMBC and selective NOE). Both ${ }^{1} \mathrm{H}$ NMR and NOE (selective band centre: $8.89 \mathrm{ppm}$ ) spectra in dmso- $d_{6}$ are shown 
With the aim of deducing the spatial arrangement of $\mathrm{H}_{2} \mathrm{~L}$ in solution, two-dimensional proton-proton (COSY) and proton-carbon (HMQC and HMBC) NMR correlation spectra, in combination with a selective NOE spectrum, have been used to (Fig. 3). The observed NOE crosspeaks between azomethine H-17 (selective band centred at 8.89 ppm), H-21 (7.58 ppm) H-1 (7.54 ppm) and H-7 (7.03 ppm) protons, which in the modelled structure are separated by 2.37, 2.10 and $2.13 \AA$, respectively, indicate that in solution is the syn isomer of $\mathrm{H}_{2} \mathrm{~L}$ with an $E$-configuration of the azomethine group.

\section{CONCLUSIONS}

The ditopic Schiff base ligand $\mathrm{H}_{2} \mathrm{~L}$ can be satisfactorily prepared and characterised. The NMR studies indicate a syn disposition for the $N, O$ donor sets of both salicylaldimine residues in solution, whereas the DFT studies indicate that this one does not appear to be the most favoured, but the barriers between the three different conformers studied are very low, and therefore very accessible for a subsequent coordination to a metal centre.

\section{EXPERIMENTAL SECTION}

$\mathbf{H}_{2}$ L. A solution of 2,6-diaminotriptycene (100 mg, $0.352 \mathrm{mmol}$ ) and 2-hydroxy-benzaldehyde (0.1 $\mathrm{mL}, 0.703 \mathrm{mmol})$ in ethanol (10 mL) with 4-5 drops of acetic acid was stirred at room temperature for overnight (reaction progress was monitored by TLC). Then, it was obtained a yellow solid, which was washed with methanol to get the desired product. Further purification was not required. Yield: above 90\%. ${ }^{1} \mathrm{H}$ NMR (400 MHz, dmso- $d_{6}$, ppm 13.05 (s, 2H, 2xOH), 8.89 (s, 2H, 2xH-17), 7.57 (d, 2H, $J=7.7, J=1.7,2 x H-21), 7.53$ (d, 2H, $J=2.1, \mathrm{H}-1$ and $\mathrm{H}-5$ ), 7.49 (d, 2H, $J=7.8, \mathrm{H}-4$ and $\mathrm{H}-8$ ), $7.44(\mathrm{~d}, 2 \mathrm{H}, J=8.6, J=2.1, \mathrm{H}-13$ and $\mathrm{H}-16), 7.35$ (t, 2H, $J=7.5, J=1.7,2 x H-19), 7.03(\mathrm{~d}, 2 \mathrm{H}, J=7.8, J=$ 2.1, H-3 and H-7), 7.00 (d, 2H, $J=8.5, J=2.3, \mathrm{H}-14$ and $\mathrm{H}-15$ ), 6.92 (t, 2H, $J=7.5, J=0.9,2 x H-20$ ), $6.90(\mathrm{~d}, 2 \mathrm{H}, J=8.3,2 \mathrm{xH}-18), 5.71$ (s, 2H, H-9 and H-10). ${ }^{13} \mathrm{C}$ NMR (125 MHz, dmso-d 6 ): $\delta / p p m 163.5$ (2xC-17), 160.6 (2xC-18a), 147.0 (2xC-10a), 145.6 (C-2, C-6), 145.2 (C-11, C-12), 144.2 (2xC-8a), 133.6 (2xC-19), 132.9 (2xC-21), 125.7 (C-14, C-15), 124.9 (C-4, C-8), 124.2 (C-13, C-16), 119.7 (2xC17a), 119.6 (2xC-20), 118.8 (C-3, C-7), 117.1 (C-1, C-5), 117.0 (2xC-18), 52.6 (C-9, C-10). IR (KBr, $\left.v / \mathrm{cm}^{-1}\right): 3442(\mathrm{OH}), 1623,1609(\mathrm{C}=\mathrm{N})$. MS (MALDI-TOF+) m/z (\%): 493.7 (100) [H $\left.{ }_{2} \mathrm{~L}+\mathrm{H}\right]^{+}$. Elemental analysis (Found): C, 82.6; H, 4.6; N, $5.6 \%$. Calcd. for $\mathrm{C}_{34} \mathrm{H}_{24} \mathrm{~N}_{2} \mathrm{O}_{2}$ (492.0) C, 82.9; $\mathrm{H}, 4.9 ; \mathrm{N}$, $5.7 \%$.

\section{Theoretical studies}

All calculations were performed using the Gaussian 09W17 program package[9] at density functional theory (DFT) level by means of the hybrid M06 functional. [10] The standard 6-31G(d) basis set was used for $\mathrm{C}, \mathrm{H}, \mathrm{O}$ and $\mathrm{N}$. To explore the conformational preference of the free ligand, the dihedral angles of the imine groups $[\mathrm{C}(2,6)-\mathrm{N}-\mathrm{C} 17-\mathrm{C} 17 \mathrm{a}]$ were progressively incremented from $0^{\circ}$ to $360^{\circ}$, and the resultant geometries were minimized using PM6, followed by a DFT single point energy calculation. The most stable conformers were further minimized by DFT. 


\section{REFERENCES}

[1] J. H. Chong, M. J. MacLachlan, Chem. Soc. Rev. 2009, 38, 3301-3315

[2] M. Mastalerz and I. M. Oppel, Angew. Chem., Int. Ed., 2012, 51, 5252

[3] C.-F. Chen, and Y.-X Ma,. Iptycene Chemistry: from Synthesis to Applications; Springer-Verlag: Berlin, 2013.

[4] S. Chakraborty, S. Mondal, N. Das, Inorg. Chim. Acta 2014, 413, 214-220

[5] S. Mondal, S. Chakraborty, S. Bhowmick, N. Das, Macromolecules 2013, 46, 6824-6831

[6] S. Chakraborty, S. Mondal, Q. Li, N. Das Tetrahedron Letters 2013, 54, 1681-1685.

[7] (a) C. J. Whiteoak, G. Salassa and A. W. Kleij, Chem. Soc. Rev., 2012, 41, 622-631; (b) K. C.; Gupta, A. K. Sutar, Coord. Chem. Rev. 2008, 252, 1420-1450

[8] IUPAC. Compendium of Chemical Terminology, 2nd ed. (the "Gold Book"). Compiled by A. D. McNaught and A. Wilkinson. Blackwell Scientific Publications, Oxford (1997). XML on-line corrected version: http://goldbook.iupac.org (2006-) created by M. Nic, J. Jirat, B. Kosata; updates compiled by A. Jenkins. doi:10.1351/goldbook.

[9] J. Frisch, G. W. Trucks, H. B. Schlegel, G. E. Scuseria, M. A. Robb, J. R. Cheeseman, G. Scalmani, V. Barone, B. Mennucci, G. A. Petersson, H. Nakatsuji, M. Caricato, X. Li, H. P. Hratchian, A. F. Izmaylov, J. Bloino, G. Zheng, J. L. Sonnenberg, M. Hada, M. Ehara, K. Toyota, R. Fukuda, J. Hasegawa, M. Ishida, T. Nakajima, Y. Honda, O. Kitao, H. Nakai, T. Vreven, J. A. Montgomery, Jr., J. E. Peralta, F. Ogliaro, M. Bearpark, J. J. Heyd, E. Brothers, K. N. Kudin, V. N. Staroverov, R. Kobayashi, J. Normand, K. Raghavachari, A. Rendell, J. C. Burant, S. S. Iyengar, J. Tomasi, M. Cossi, N. Rega, J. M. Millam, M. Klene, J. E. Knox, J. B. Cross, V. Bakken, C. Adamo, J. Jaramillo, R. Gomperts, R. E. Stratmann, O. Yazyev, A. J. Austin, R. Cammi, C. Pomelli, J. W. Ochterski, R. L. Martin, K. Morokuma, V. G. Zakrzewski, G. A. Voth, P. Salvador, J. J. Dannenberg, S. Dapprich, A. D. Daniels, Ö. Farkas, J. B. Foresman, J. V. Ortiz, J. Cioslowski, and D. J. Fox, GAUSSIAN 09, (Revision A.2), Gaussian, Inc., Wallingford CT, 2009.

[10] (a) Y. Zhao, D. G.Truhlar, Theor. Chem. Acc. 2008, 120, 215-241. (b) Y. Zhao, D. G.Truhlar, Acc. Chem. Res. 2008, 41, 157-167. 